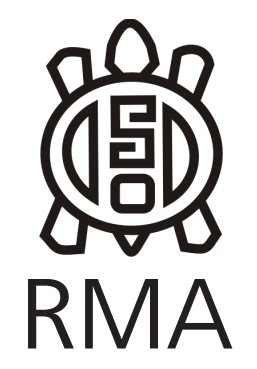

Editorial

\title{
Editorial
}

Gabriela Roxana Cattáneo*

*Instituto de Antropología de Córdoba, IDACOR, CONICET, Universidad, Nacional de Córdoba, Córdoba, Argentina, **Universidad Nacional de Córdoba, Facultad de Filosofía y Humanidades, Museo de Antropología, Córdoba, Argentina.

E-mail: rcattaneo@ffyh.unc.edu.ar

Es con mucha alegría y orgullo que presentamos este volumen especial de la Revista del Museo de Antropología que reúne una parte de los trabajos presentados durante el $1^{\circ}$ Congreso Argentino de Estudios Líticos en Arqueología realizado desde el 19 al 22 de septiembre de 2018 en la ciudad de Córdoba y realizado en el predio del CIECS (CONICETUNC).

Dicho evento contó con el financiamiento de diversas instituciones: CONICET RC 2018, MINCYT (Prov. de Córdoba), FONCYT (RC2018 0152), IDACOR (CONICET-UNC) y SECYT de la Universidad Nacional de Córdoba. Asimismo fueron varias las Entidades Auspiciantes, que nuclean a la gran mayoría de los investigadores en estas temáticas:

- Asociación de Arqueólogos Profesionales de la República Argentina (AAPRA)

- Sociedad Argentina de Antropología (SAA)

- Centro de Estudios en Arqueología Histórica, Facultad de Humanidades y Artes. Universidad Nacional de Rosario.

- Laboratorio de Arqueología y Etnohistoria, Departamento de Historia, Facultad de Ciencias Humanas, Universidad Nacional de Río Cuarto.

- Centro de Investigaciones Precolombinas Instituto Superior del Profesorado Dr. Joaquín V. González - Dirección de Educación Superior - Gobierno de la Ciudad Autónoma de Buenos Aires.

- Instituto de Antropología y Pensamiento Latinoamericano. Ministerio de Cultura. Presidencia de la Nación.

- Escuela de Arqueología (UNCA)

- CADIC-CONICET

- Instituto Multidisciplinario de Historia y Ciencias

\section{Humanas. CONICET.}

- Facultad de Ciencias Exactas y Naturales, Universidad Nacional de Cuyo.

- Facultad de Cs. Físicas, Matemáticas y Naturales de la Universidad Nacional San Luis, Dpto. de Geología.

- Instituto Superior de Estudios Sociales (CONICET-UNT).

- Instituto de Arqueología y Museo (IAM), Universidad Nacional de Tucumán.

- Facultad de Filosofía y Humanidades, Universidad Nacional de Córdoba

- Instituto de Antropología de Córdoba (IDACOR/ CONICET- Universidad Nacional de Córdoba)

- Laboratorio de Análisis Macro y Microscópico de Materiales Líticos (LAMMAL IDACOR-Museo de Antropología, CONICET/UNC)

- Programa de Arqueología Digital (IDACORMuseo de Antropología, CONICET/UNC)

- Facultad de Ciencias Naturales y Museo, Universidad Nacional de La Plata.

El $1^{\circ}$ Congreso Argentino de Estudios Líticos en Arqueología ( $1^{\circ}$ CAELA) fue un evento de carácter nacional que se realizó por primera vez en Argentina, siendo único en Sudamérica en su tipo. Dado ese carácter, la convocatoria fue de interés macroregional, sumando los países del Cono Sur donde también existen especialistas en estudios líticos que no poseen reuniones sobre este tópico o área de investigación.

Fueron presentados en 10 simposios más de 100 trabajos, de autores nacionales de casi todas las provincias de Argentina y de Brasil, Chile, Uruguay, Perú y Estados Unidos. 
Se contó con la participación del Dr. Bruce Bradley (University of Exeter, UK), el arq. Wilfredo Faundes Catalán (Universidad de Tarapacá, Chile), y las especialistas argentinas Dra. Patricia Escola (CITCA, CONICET-UNCA), Cristina Bellelli (CONICET-INAPL), Nora Flegenheimer (CONICET-Área Arqueología y Antropología, Museo de Ciencias Naturales de Necochea), el invest. Carlos Aschero (ISES CONICET) y el Dr. Marcelo Cardillo (IMICIHU CONICETUBA) (quienes dictaron talleres, conferencias y conversatorios (ver más adelante).

Dentro de las actividades académicas desarrolladas durante la reunión los Simposios fueron:

1. Arqueologia Experimental. Coordinadores: Hernán H. De Angelis (CADIC-CONICET), Damián Bozzuto (CONICET-INAPL), y Celeste Weitzel (CONICET- AyA, Área de Museos, Municipalidad de Necochea)

2. El estudio de conjuntos líticos en las áreas de aprovisionamiento: variabilidad y formas de abordaje desde el análisis técnico-tipológico. Coordinadores: Federico Bobillo (ISES-CONICET, Tucumán), María Laura Salgán (IANIGLA-CONICET Grupo Vinculado de San Rafael) y Gisela Sario (IDACOR-CONICET y Museo de Antropología, FFyH, UNC)

3. Análisis de la explotación del volumen de las rocas. Coordinadores: Paula Barros y Florencia Santos Valero (Instituto INCUAPA-CONICET, Facultad de Ciencias Sociales. Universidad Nacional del Centro de la Provincia de Buenos Aires). Relator: Guillermo Heider (CONICET - Departamento de Geología, Facultad de Ciencias Físico, Matemáticas y Naturales, Universidad Nacional de San Luís)

4. Desde las fuentes: avances en los estudios de selección y aprovisionamiento de rocas y sus implicancias en las prácticas sociales Coordinadores: Fabiana Skarbun y Manuel E. Cueto. Relator: Ariel D. Frank (División Arqueología. Facultad de Ciencias Naturales y Museo de La Plata / CONICET)

5. Avances y desafíos en torno a los abordajes cuali-cuantitativos aplicados a los estudios líticos. Coordinadores: Marcelo Cardillo (IMHICIHUCONICET) y Federico Restifo (ICSOH Universidad Nacional de Salta)

6. Perspectivas de análisis integral en el estudio de artefactos líticos. Coordinadores: Estela Mansur
(CADIC CONICET), Patricia Escola (CITCA-CONICETUNCAT), Salomón Hocsman (ISES-CONICET-UNT) y María del Pilar Babot (ISES-CONICET-UNT)

7. El estudio de los procesos de formación del registro arqueológico y sus efectos en los conjuntos líticos. Coordinadores: Eugenia Carranza (CONICETIMHICIHU), Catalina Baliran (FFyL- UBA) y Federico Bobillo (Instituto de Arqueología y Museo (ISESCONICET), UNT. San Miguel de Tucumán). Relatora: Karen Borrazzo (CONICET-IMHICIHU y FFyL- UBA)

8. Hacia la creación de repositorios digitales con bases de datos de información sobre materias primas líticas en arqueología. Coordinadoras: Nora Flegenheimer (CONICET-Municipalidad de Necochea), Roxana Cattáneo (IDACOR-CONICET y FFyH, UNC), Natalia Mazzia (CONICET-Municipalidad de Necochea)

Además, una Mesa de discusión:

9. La conservación preventiva de objetos y estructuras líticas, casos de aplicación, teoría y metodología. Coordinadores: Paola Geovanna Maschio (Facultad de Arquitectura, Urbanismo y Diseño-Universidad Nacional de Córdoba) y Eduardo A. Pautassi (IDACOR-CONICET y FFyH, UNC)

Y, por último, una Mesa de Estudiantes.

10. Temas Generales. Coordinadores: Camila Brizuela (Lic. en Antropología, FFyH, UNC), Florencia Costantino (Lic. en Antropología, FFyH, UNC y Maximiliano Córdoba Barrera (Lic. en Antropología, FFyH, UNC)

De cada uno de estos Simposios el lector podrá encontrar una excelente representación de las ponencias en los 47 trabajos que conforman este volumen. Cada Simposio cuenta además con un prólogo de los Coordinadores que fueron invitados a colaborar con nuestra Revista como Editores invitados, estando encargados a través de nuestro sistema OJS de realizar el proceso de evaluación. En el caso de los Editores invitados estuvo bajo mi responsabilidad el proceso editorial para respetar las evaluaciones anónimas. Un especial agradecimiento por todo el esfuerzo, el trabajo y la seriedad con la que llevaron esta tarea.

Pero el Congreso contó además con actividades que 
complementaron las sesiones:

a.- Presentación de estado de situación de la nueva versión de "Ensayo para una clasificación morfológica de artefactos líticos aplicada a estudios tipológicos comparativos" y "Ensayo para una clasificación morfológica de los artefactos líticos. Apéndices A y B."

b.- un Curso de Postgrado del Doctorado en Cs. Antropológicas de la FFYH-UNC y el Doctorado en Arqueología de la UNT, dictado por el Dr. Bruce Bradley (University of Exeter, UK) con la colaboración de la Lic. Nora Flegenheimer (CONICETMunicipalidad de Necochea), a ser dictado durante los tres días anteriores al inicio del Congreso. Con cupo.

c.- Un taller denominado "Introducción al análisis cuantitativo de la forma en materiales líticos" a ser dictado por el Dr. M. Cardillo (IMHICIHU-CONICET). Con cupo.
d.-Un viaje de 1 día de duración a sitios arqueológicos de la prov. de Córdoba, al finalizar el evento el sábado 22 de Septiembre. Con una visita de sitios arqueológicos del valle de Ongamira Alero Deodoro Roca y la visita al Museo Deodoro Roca, donde se compartió un almuerzo tradicional.

Se sumaron además las Conferencias de especialistas invitados:

a.- Conferencia B. Bradley y D. Stanford - Rocking the Boat; Flaked Stone Assemblages as Indicators of Historical Connections: the case of the Solutrean Hypothesis (Lugar: Museo de Antropología, Av. Hipólito Yrigoyen 174)

b.- Conferencia Inaugural de C. Aschero "Propuesta y perspectivas de una tipología morfológica" (Lugar: Museo de Antropología, FFyH-UNC, Av. Hipólito Yrigoyen 174)

c.- Conversatorio: Una historia en construcción, los

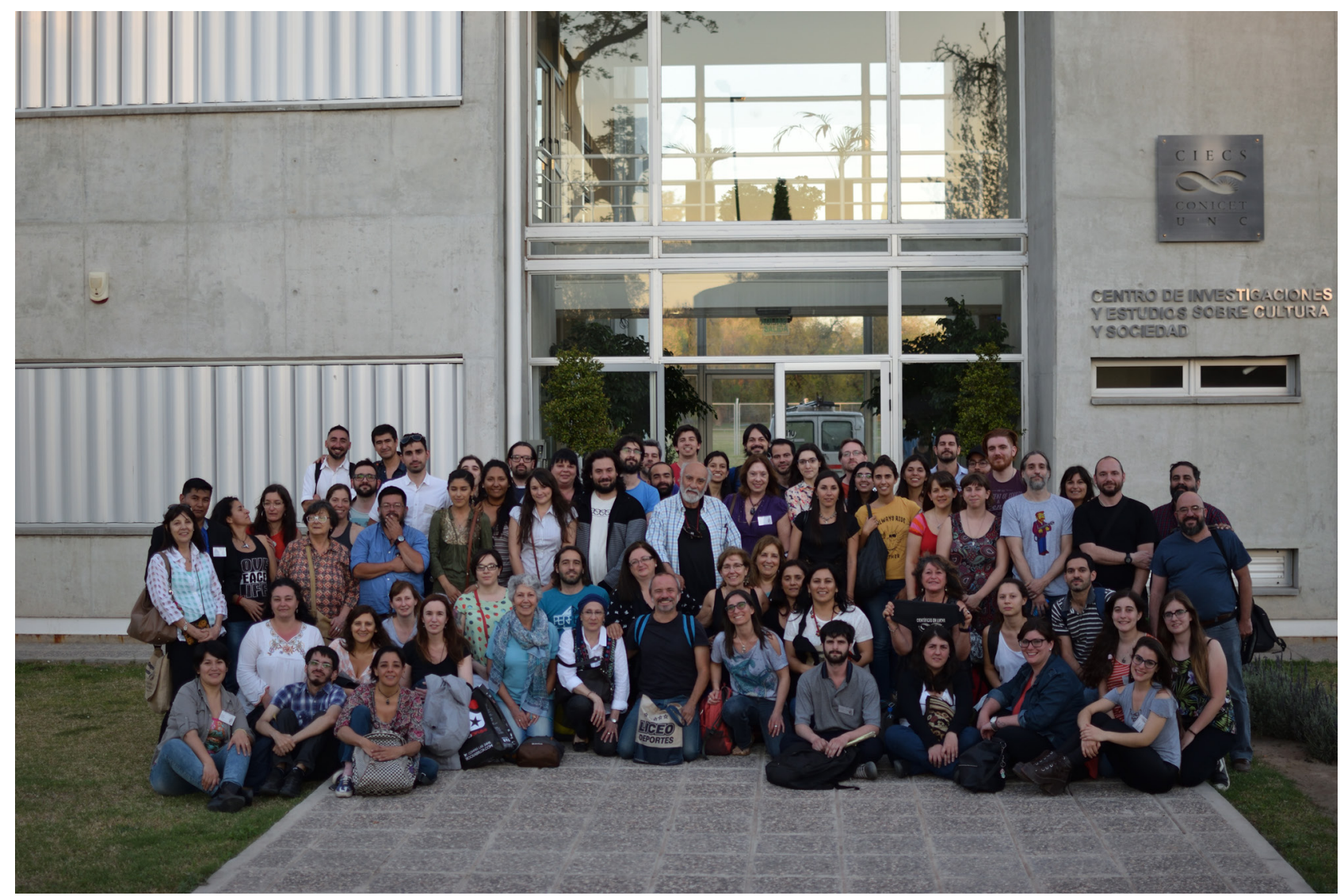

Figura 1. Participantes del 1 CAELA al finalizar el último día de sesiones.

Figure 1. Participants of 1 CAELA at the end of the last day of sessions. 
estudios líticos en Argentina.

A 10 años de "La Arqueología y las Piedras "C. Bellelli y N. Flegenheimer. (Lugar: CIECS CONICETUNC).

f.- Una Feria de materias primas. Se contó con un espacio acondicionado para realizar muestras e intercambio de rocas, experiencias de talla, muestras de materiales.

g. Actividad libre de arquería. Sin inscripción, ni cupo. Profesores de la escuela de Arquería de Córdoba "Krummi Krunkar" y los arqueólogos Dres. M. Colombo y D. Bozzuto prepararan un sector del predio acondicionado para la realización de lanzamientos intuitivos de arco y flecha/lanzas/ hachas, abierto a todo el público asistente.

En relación a la cantidad de participantes se contó con alrededor de 200 participantes distribuidos en todas las actividades (Figura 1). A continuación se presentan la cantidad de trabajos presentados en dos formatos, por un lado de acuerdo al origen geográfico del lugar de trabajo de los autores (institutos, universidades, etc.) para mostrar desde donde han venido a presentar sus trabajos los asistentes al Congreso (Tabla 1) y por otro la provincia o tema de interés de las ponencias a los fines de mostrar el interés espacial de los estudios líticos de los cuales se presentaron trabajos (Tabla 2).

\section{En cuanto a la difusión de los resultados.}

Los resultados del Congreso se difundieron por diversos medios gráficos y digitales. En el primer caso se distribuyeron en formato papel el Libro de Resúmenes y el cronograma de las actividades y ponencias, el mismo puede ser descargado en la actualidad de una Comunidad virtual situada en el

Tabla 1. Procedencia de los autores que participaron del 1 CAELA.

Table 1. Provenience of the authors who participated in the 1 CAELA.

\begin{tabular}{lcr}
\hline Procedencia geográfica & Cantidad de autores & $\%$ \\
\hline Buenos Aires (CABA, La Plata, Necochea, Mar del Plata, & 120 & 44.44 \\
Olavarría) & 38 & 14.07 \\
Córdoba & 20 & 7.41 \\
Tucumán & 15 & 5.56 \\
Mendoza & 11 & 4.07 \\
Tierra del Fuego & 9 & 3.33 \\
San Luis & 8 & 2.96 \\
Catamarca & 6 & 2.22 \\
Salta & 6.85 \\
Santa Fe & 5 & 1.48 \\
Chubut & 4 & 1.11 \\
Jujuy & 3 & 0.74 \\
Río Negro & 2 & 0.37 \\
Santa Cruz & 1 & \\
Estados Unidos & & 4.44 \\
Brasil & 12 & 2.59 \\
Chile & 7 & 1.85 \\
Francia & 7 & 0.74 \\
Italia & 5 & 0.37 \\
Perú & 2 & 0.37 \\
Total & 1 & 100.00 \\
\hline
\end{tabular}


Repositorio Suquía (IDACOR-CONICET-UNC) en el siguiente link https://suquia.ffyh.unc.edu.ar/handle/ suquia/2939.

Por otro lado se crearon dos endpoints en formato web. El primero en formato Blog que integró novedades y anuncios. Durante el Congreso sirvió como punto de acceso a los resúmenes y cronogramas. Este se enlazó con el segundo, que consistió en una instalación de Open Conference System, un software que garantiza la publicación y preservación de los resúmenes, a la vez que su visualización web. Todo esto fue cosechado por el Repositorio Suquía. Todos estos sistemas permiten el acceso libre a la documentación generada y su preservación digital.

La posibilidad de presentar nuestras ideas y resultados, encontrarnos, re-conocernos, charlar, compartir momentos, y que todo haya sido en un ámbito de extrema cordialidad se dio gracias a cada uno de los participantes del evento y de un nutrido grupo de graduados y estudiantes que colaboraron en la Comisión Organizadora Local y en una Comisión Organizadora más grande, con representantes de todas las regiones de Argentina ¡A todos ellos nuestro agradecimiento!

El encuentro fue fructífero, e incluyó un homenaje a las docentes del Curso de Talla, Nora Flegenheimer, Patricia Escola y Cristina Bayón, que a lo largo de los años formaron a decenas de colegas en el estudio de los materiales líticos a través de sus enseñanzas de la talla experimental. Aquí también va nuestro reconocimiento a todos los colegas y familiares que apoyaron la realización de este momento.

La actividad anterior fue el preludio para la fiesta de cierre que se desarrolló en el Museo de Antropología (FFyH-UNC).

Tabla 2. Cobertura geográfica de los estudios líticos que se presentaron en trabajos del 1 CAELA.

Table 2. Geographical coverage of the lithic studies that were presented in works of the 1 CAELA.

\begin{tabular}{lcc}
\hline Procedencia geográfica & Casos & $\%$ \\
\hline Córdoba & 41 & 17.23 \\
Santa Cruz & 40 & 16.81 \\
Buenos Aires (CABA, La Plata, Necochea, Mar del Plata, Olavarría) & 28 & 11.76 \\
Catamarca & 17 & 7.14 \\
Mendoza & 12 & 5.04 \\
Neuquén & 12 & 5.04 \\
Río Negro & 10 & 4.20 \\
San Juan & 10 & 4.20 \\
Salta & 8 & 3.36 \\
Tierra del Fuego & 8 & 3.36 \\
Tucumán & 8 & 3.36 \\
Brasil & 7 & 2.94 \\
Jujuy & 6 & 2.52 \\
La Pampa & 5 & 2.10 \\
San Luis & 5 & 2.10 \\
Chile & 5 & 2.10 \\
Chubut & 2 & 0.84 \\
Entre Ríos & 3 & 1.26 \\
Perú & 1 & 0.42 \\
Experimental/conceptual & & \\
Total & 10 & 4.20 \\
\hline
\end{tabular}


Finalmente, el último día se celebró la Asamblea final donde se eligió a Cristina Bellelli (CONICETINAPL) como la presidenta del próximo Congreso y la ciudad de Bs As como el lugar para llevarlo a cabo. Esperando ansiosos y ansiosas este nuevo encuentro quiero cerrar este prólogo con un gran agradecimiento a todos y todas, los y las colegas del IDACOR- CONICET (Dr. Andrés Laguens) y del
Museo de Antropología (Mgter. Fabiola Heredia) por el éxito de la reunión. Ahora los invito a disfrutar y compartir la lectura que nos encuentra en un momento especial de la historia, la cuarentena por el COVID 19, un virus de la gripe que se ha convertido en Pandemia mundial que nos mantiene en nuestras casas. Un abrazo fraterno.

Córdoba, 30 de marzo de 2020 